\title{
Clinical significance of ESBL-producing Escherichia coli isolated from urinary tract infections in sylhet Bangladesh
}

\begin{abstract}
The prevalence of extended-spectrum b-lactamases (ESBL) producing E. coli in the town of Sylhet was assessed over 12months period. One hundred patients who had symptomatic urinary tract infections were selected for this study and urine samples were collected from the private laboratories of the community. These isolates were further confirmed as ESBL-producing E. coli by phenotypic methods were identified and selected. Patients having urinary tract infection showed $72 \%$ were female and the rest $28 \%$ were male. The investigations were carried out on female population only. The patient's age ranged from 4 years to 60years. The highest age incidence of UTI patient was 21-30years (28\%) followed by $31-40$ years $(20 \%)$. Patients in the age range $41-50$ years showed $15 \%$ and patients above 50 years $19 \%$. The antibiotic susceptibility test for non-ESBL showed $100 \%$ sensitivity to imipenem and meropenem. There was multidrug resistance to amoxicillin-clavulanic acid $30 \%$ ceftazidime $40 \%$, ceftriaxone $35 \%$, and ciprofloxacin $60 \%$. The ESBL group was also $100 \%$ sensitive to carbapenems and the rest of resistance by percentage were amoxicillinclavulanic acid $-80 \%$, ceftazidime- $55 \%$, ceftriaxone- $80 \%$, ciprofloxacin $80 \%$. The ESBL and non ESBL producing strains were separated for plasmid profile analysis. The plasmid profile showed $60 \%$ of isolates exhibit high molecular weight plasmids ( $>140 \mathrm{MDa}$ ) in ESBL groups and similar results were seen in Non ESBL groups (64\%). These findings suggests that both ESBL and non ESBL producing isolates harbour large size plasmids, despite the fact that ESBL-producing strains cause wide range of multi-drug resistance in the community.
\end{abstract}

Keywords: uropathogenic E.coli, ESBL E.coli, UTI, Echerichia coli
Volume 4 Issue 6 - 2017

\author{
Belal Mahmood,' Sarah Furrows, ${ }^{2}$ Shaheen \\ Methar, ${ }^{2}$ Mohammed Abul Hasnat, ${ }^{3}$ Kamrul \\ Hassan ${ }^{3}$ \\ 'Head of Medical Microbiology, North East Medical College, \\ Bangladesh \\ ${ }^{2}$ Stellenbosch University, South Africa \\ ${ }^{3}$ Kingston Hospital Surrey, UK University Hospital of Leicester, \\ UK
}

Correspondence: Belal Mahmood, Department of Microbiology, North East Medical College, Sylhet, Bangladesh, Email belalm1958@gmail.com

Received: June 03, 2017| Published: June 19, 2017

\section{Introduction}

Urinary tract infections (UTI) are a serious health problem affecting millions of people each year. UTIs have been reported to affect up to 150 million individuals annually world wide. ${ }^{1}$ They are the most common bacterial infection in humans and account for significant morbidity and health care cost. Infections of the urinary tract are the second most common type of infection in the human infections and are mainly caused by uropathogenic E. coli. These bacteria are responsible for between $70 \%$ to $90 \%$, of community-acquired UTI. ${ }^{2}$ They contribute largely (38-40\%) to nosocomial infections. ${ }^{3}$ The severity of UTI depends both on the virulence of the bacteria and the susceptibility of the host.

Recently ESBL-producing organisms have been reported from many parts of the world. ${ }^{4,5}$ In Bangladesh a preliminary study showed that $E$. coli can be isolated from different sources such as urine, stool, etc. ${ }^{6}$ The significance of ESBL is that ESBL producing organisms are resistant to second and third generation cephalosporins, because the extended spectrum b-lactamases hydrolyse the $\beta$-lactam ring of the antibiotics. As a result, these antibiotics become resistant and ineffective to bacteria.

This study was therefore designed to reveal the clinical significance of UTI patients caused by ESBL- producing E. coli and their relationship with molecular characterizations.

\section{Materials and methods}

\section{Study population and types of specimen}

The study period was for 12 months. Urine samples were collected from patients having clinical manifestations of urinary tract infection. The age and sex of the patients were recorded. All the samples were collected from outpatient's departments and private laboratories. The specimen type included in this study was urine collected from patients by clean catch mid stream urine.

\section{Microbiological methods}

All samples were routinely cultured on MacConkey agar. Cultures were investigated by biochemical reactions such as Kligler Iron Agar (KIA), indole, urease, and citrate utilization. Microscopic examination of urine for pus cells was performed in each sample of urine. Pus cells more than five were considered significant.

\section{Antibiotic susceptibility testing}

Antimicrobial susceptibility testing of the isolated organisms was done by a disk diffusion method using the Kirby-Bauer technique as recommended by NCCLS. All disks were obtained from Oxoid Ltd., Basingstoke, Hampshire, UK. Antibiotic potency of the disks was standardized against the reference strains, E. coli ATCC 25922. 


\section{Detection of ESBL by double disk diffusion synergy method}

ESBL was detected by double disk synergy technique in which an Augmentin disk (amoxicillin 20 $\mu \mathrm{g}$ and clavulanic acid $10 \mu \mathrm{g}$ ) was placed in the centre of a plate and cefotaxime $(30 \mu \mathrm{g})$, ceftazidime $(30 \mu \mathrm{g})$, aztreonam $(30 \mu \mathrm{g})$ and ceftriaxone $(30 \mu \mathrm{g})$ disk were placed $30 \mathrm{~mm}$ (centre to centre) from the Augmentin disk. The enhancement of zone of inhibition of any one of the four drug disk towards the disk containing clavulanic acid suggested the presence of an extendedspectrum $\beta$-lactamase.

\section{Separation and isolation of plasmid DNA by agarose gel electrophoresis}

An isolated colony of each isolate was inoculated into $1.5 \mathrm{ml}$ of TSB broth with $0.3 \%$ yeast extract (YE) and incubated overnight at $37^{\circ} \mathrm{C}$ on a water bath shaker. Cells were collected in an eppendorf tube and centrifuged for $5 \mathrm{~min}$ at $3000 \mathrm{rpm}$. The supernatant was removed and the pellets were suspended in Kado sol-1, Kado sol2 and finally phenol was added. The suspension was mixed till it became a milky white suspension. Then the tubes were centrifuged at room temperature for 8 minutes. Using a pipette the plasmid solution was removed carefully and transferred into new eppendorf tubes. Plasmid DNA was separated by horizontal electrophoresis in $0.7 \%$ agarose slab gels in a tris-borate EDTA buffer at room temperature at 100 volt $(50 \mathrm{~mA})$ for 3 hours. Briefly, $30 \mu 1$ of plasmid DNA solution was mixed with $3 \mu 1$ of tracking dye (Appendix 11) and was loaded into the individual well of the gel. The gel $5 \mathrm{~mm}$ thick was stained with $0.5 \mu \mathrm{g} / \mathrm{ml}$ of ethidium bromide for $30 \mathrm{~mm}$ at room temperature. DNA bands were visualized and photograph was taken using an MP4 land camera with UV transilluminator.

\section{Results}

\section{Plasmid profile analysis of E.coli isolates}

Analysis of Plasmid DNA by agarose gel electrophoresis revealed that all the isolates contained multiple numbers of plasmid ranging from 1 to $140 \mathrm{MDa}$, forming a unique banding pattern. High molecular weight 140MDa plasmids were found to be present in majority isolates $(69.2 \%)$ and remaining isolates harboured $30-90 \mathrm{MDa}$ plasmid. Plasmids patterns were formed according to the number and size of the plasmid and each isolate was grouped into a particular pattern (Figures 1-5).

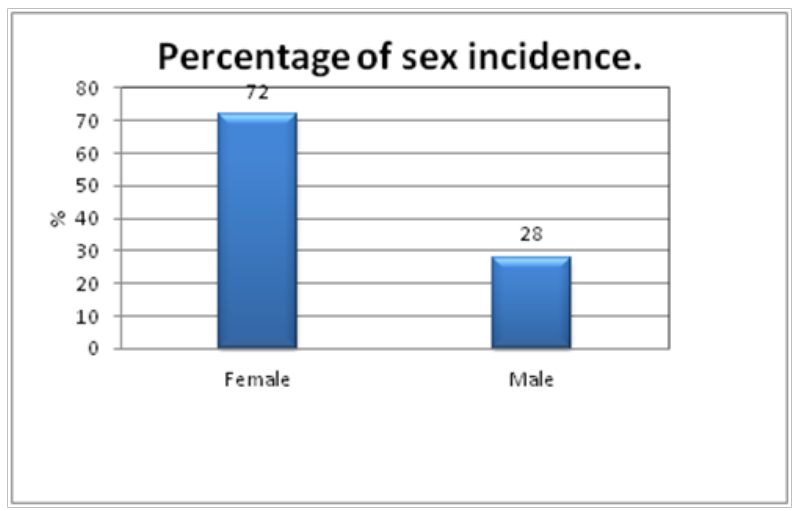

Figure I Showed the percentage distribution of male and female populations suffering from Urinary Tract Infections. The table clearly indicates the incidence of female subjects were higher (72\%) compared to male subjects $(28 \%)$. Confidence interval for female population is $56.2 \%$ to $82.2 \%(P=0.0005)$.

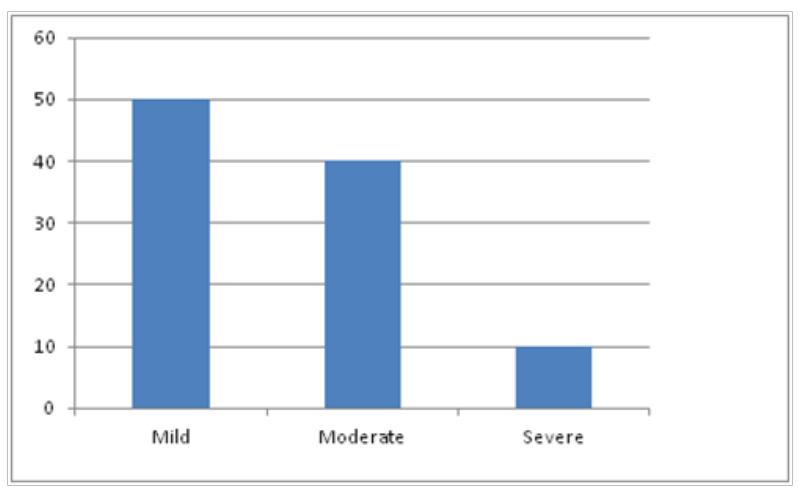

Figure 2 Showed that the patients having bacterial urine culture $\sim 105 \mathrm{CFU} /$ $\mathrm{ml}$ associated with 5-10 microscopic urine leukocytes were designated as mild symptoms whereas patients having bacterial culture $>10^{5} \mathrm{CFU} / \mathrm{ml}$, associated with microscopic urine leukocyte 10 to 15 were designated as moderate symptoms and the patients having bacterial culture $>105 \mathrm{CFU} / \mathrm{ml}$, associated with microscopic leukocyte in urine numerous, were grouped as severe symptomatic UTI patients.

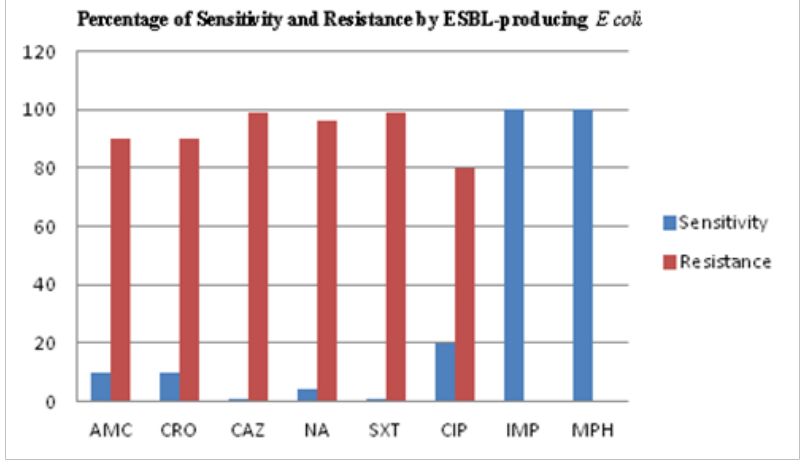

Figure 3 Antibiogram of ESBL E.coli isolates from symptomatic UTI patients.

The results show that the total number of ESBL isolates were 20. All isolates were sensitive to meropenem and imipenem; $90 \%$ were resistant to amoxicillin-clavulanic acid; $95 \%$ were resistant to ceftazidime; $90 \%$ were resistant to ceftriaxone; $80 \%$ were resistant to ciprofloxacin.

AMC, amoxicillin-clavulanic acid; CAZ, ceftazidime; CRO, ceftriaxone; CIP ciprofloxacin; IPM, imipenem

MPM, meropenem

1415161718 M19202122 23242526 M

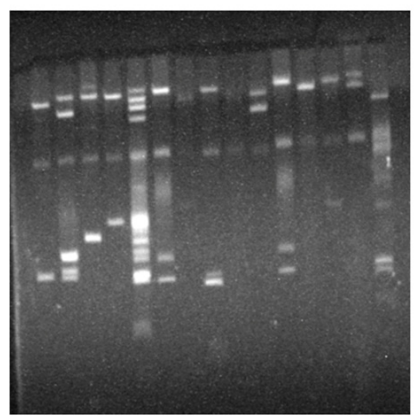

Figure 4 Agarose gel electrophoresis of plasmid DNA showing representative of different ESBL E.coli Isolates. Analysis of the plasmid DNA by Agarose gel electrophoresis showed that the isolates contained multiple numbers of plasmids ranging from I.0 to I40MDa, which formed a unique banding pattern in many of these isolates). CHR indicates the banding position of the chromosomal DNA. 
$\begin{array}{lllllllllllllll}M & 1 & 2 & 3 & 4 & 5 & 6 & 7 & 8 & 9 & 10 & 11 & 12 & 13 & \mathrm{M}\end{array}$

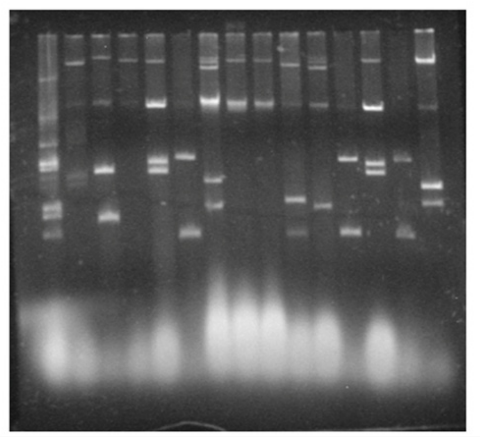

Figure 5 Agarose gel electrophoresis of plasmid DNA showing representative of different Non ESBL E.coli Isolates. Analysis of the plasmid DNA by Agarose gel electrophoresis showed that the isolates contained multiple numbers of plasmids ranging from I.0 to I40MDa, which formed a unique banding pattern in many of these isolates. CHR indicates the banding position of the chromosomal DNA.

Chi square test was used to analyze the data statistically. Epi Info version 5 was used for this analysis. Patients with mild symptoms were having higher prevalence than patients with severe symptoms $(\mathrm{p}<0 \cdot 001)$

\section{Discussion}

There has been limited study on ESBL- producing organisms in Bangladesh therefore this protocol was undertaken to highlight the clinical importance of extended spectrum beta- lactamase producing Escherichia coli isolated from patients having community-acquired urinary tract infections.

The first reported study was conducted in Dhaka city. ${ }^{6-8}$ Samples were collected from various sources such as urine sputum, pus, throat swab, etc. Urine samples showed the highest incidence of ESBL production. ${ }^{6}$ The present study clearly showed that out of 77 E.coli strains, only 20 strains were documented to be ESBL phenotypes.

The current investigation further revealed $72 \%$ female populations were having UTI and were free from pregnancy, cystitis and pyelonephritis. Therefore the present study was carried out on uncomplicated cases. Serogrouping of each ESBL isolate showed results similar to other studies. ${ }^{2}$

The sensitivity profile in previous studies indicated that majority of the strains were resistant to commonly used antibiotics. Several studies in the past have documented these findings. ${ }^{1}$ This is based on the fact that the $\beta$-lactamase produced by E.coli; has hydrolytic effects on antibiotics having $\beta$ - lactam ring. ${ }^{9}$ As a result these antibiotics become ineffective and the patients continue to suffer for a long time.

In the present study, drugs like amoxicillin-clavulanic acid, ceftazidime, ceftriaxone and ciprofloxacillin showed higher incidence of resistance in ESBL isolates. In Non ESBL isolates the sensitivity percentage was higher compared to ESBL groups. New drugs like imipenem and meropenem were still showing $100 \%$ sensitivity with no evidence of resistance so far.

These results suggested that ESBL UTI patients having Escherichia coli are frequently exposed to antibiotics and gradually developed multi drug resistance..$^{5,10}$ The majority of the patients in present study had history of antibiotic intake. Therefore it was speculated that these isolates undergo plasmids or chromosome mediated resistance, in the community.
The investigation was further carried out for plasmid profile only. It revealed that the majority of ESBL isolates were having multidrug resistance factor. The isolates also harboured high molecular wt plasmids (Molecular wt $\geq 140 \mathrm{MDa}$ ) both for ESBL $(60 \%)$ and non ESBL isolates (64\%. Plasmids of high mol. wt are generally said to be transmissible between the organisms by the process of conjugation. ${ }^{4}$

These results also suggest that uropathogenic E.coli were probably originating from faecal contamination from unhygienic perineum. Previous studies have documented that high molecular wt plasmids (140MDa) are present in enteropathogenic E.coli as well (10) and ESBL E.coli have been detected in stool as well. ${ }^{11}$ It is a matter of controversy if these uropathogens were originating from diarrhoeagenic E.coli.

Therefore the present study needs further work on identification of genes responsible for the synthesis of ESBL. Studies in the past have documented that isolates with ESBL phenotypes contained blaTEM, blaSHV, blaOXA, and blaCTX-M genes which were confirmed by polymerase chain reactions. ${ }^{4,11-19}$

\section{Conclusion}

The results distinctly showed that ESBL strains are emerging in a town like Sylhet and these strains were responsible for plasmid mediated multidrug resistance. In a developing country like Bangladesh there is indiscriminate use of antibiotics. As a result the treatment strategy is getting complicated and the therapeutic options are limited. Therefore more molecular research is required to understand the mechanisms of drug resistance.

The ESBL uropathogenic strains were responsible for plasmid mediated multidrug resistance in the community of Sylhet region. These isolates also harbour large sized plasmids which are transmissible in the community.

\section{Acknowledgements}

I am indebted to Dr. Kaisar Ali Talukdar for the financial support to carry out this investigation on urinary tract infection project at International Centre for Diarrhoeal Diseases \& Research Mohakhali Dhaka Bangladesh. I am also grateful to Kamrul Hasan for collecting the samples from remote areas of Sylhet regions.

\section{Conflict of interest}

The author declares no conflict of interest.

\section{References}

1. Aboderin OA1, Abdu AR, Odetoyin BW, et al. Antimicrobial Resistance in Escherichia coli strains from Urinary Tract Infections. J Natl Med Assoc. 2009;101(12):1268-1273.

2. Li D1, Liu B, Chen M, et al. A multiplex PCR method to detect 14 Echerichia coli serogroups associated with urinary tract infections. $J$ Microbiol Methods. 2010;82(1):71-77.

3. Struelens MJ, Denis O, Rodrigues-Villalobos H. Microbiology of nosocomial infections: progress and challenges. Microbes Infect. 2004;6(11):1043-1048.

4. Jacoby GA, Sutton LA. Properties of Plasmids responsible for production of Extended-Spectrum $\beta$-Lactamases. Antimicrob Agents Chemother. 1991;35(1):164-169.

5. Jadhav S, Hussien A, Devi S, et al. Virulence Characteristics and Genetic Affinities of Multiple Drug Resistant. Uropathogenic Escherichia coli from a Semi Urban Locality in India. Plos One. 2011;6(3):e18063. 
6. Rahman MM, Haq A, Hossain MK, et al. Prevalence of extendedspectrum $\beta$-lactamases-producing Escherichia coli and Klebsiella pneumoniae in an urban hospital in Dhaka, Bangladesh. Int J Antimicrob Agents. 2004;24(5):508-510.

7. Rahman NMW, Lutfor AB, Jhora ST, et al. Detection of CTX-M gene in extended spectrum beta lactamase (ESBL) producing Escherichia coli and Klebsiella species of different hospitals. Bangladesh J Med Microbiol. 2010;4(2):28-31.

8. Rahman NMW, Lutfor AB, Jhora ST, et al. Transfer of antibiotic gene CTX-M- An alarm for the clinician. Bangladesh $J$ Med Microbiol. 2011;5(0):2-5.

9. Bradford PA. Extended-spectrum beta-Lactamases in the 21 st century characterization epidemiology, and detection of this important resistance threat. Clin Microbiol Rev. 2001;14(4):933-951.

10. Chowdhury MAQ, Rahman KM, Miah MRA, et al. Transferable drug resistnce (R-factor) among the Enterobacteriaceae in urinary tract infections: a study at an urban hospital in Bangladesh. J Trop Med Hyg. 1994;97(3):161-167.

11. Marijan T, Jasmina Vrane J, Bedeni B, et al. Emergence of uropathogenic extended-spectrum $\beta$ lactamases-producing Escherichia coli strains in the community. Coll Antropol. 2007;31(1):227-233.

12. Lina TT, Khajanchi BK, Azmi IJ, et al. Phenotypic and Molecular Characterization of Extended Spectrum Beta-Lactamase-Producing Escherichia coli in Bangladesh. PloS One. 2014;9(10):e108735.
13. Hoque N, Kibria G, Begum SN. Prevalence of urinary tract infection in adult women. Bangladesh Med Res Counc Bull. 1976;11(2):75-85.

14. Karlowsky JA, Kelly LJ, Thomsberry C, et al. Trends in antimicrobial resistance among urinary tract infections of Escherichia coli from female outpatients in the United States. Antimicrob Agents Chemother. 2002;46(8):2540-2545.

15. Mohammed A, Mohammed S, Khan AU. Etiology and antibiotic resistance patterns in J N N C Hospital Aligarh, India. Ann Clin Microbiol Antimicrob. 2007;6:4.

16. Munday CJ, Xiong J, Li C, et al. Dissemination of CTX-M type $\beta$ lactamases in Enterobacteriaceae isolates in the People's Republic of China. Int J Antimicrob Agents. 2004;23(2):175-180.

17. Peirano G, Cornelius HJ, Greune van, et al. Characteristics of infections caused by extended-spectrum $\beta$-lactamase-producing Escherichia coli from community hospital in South Africa. Diagn Microbiol Infect Dis. 2011;69(4):449-453.

18. Suzuki S, Shibata N, Yamane K, et al. Change in the prevalence of extended-spectrum-lactamases producing Esherichia coli in Japan by clonal spread. J Antimicrob Chemother. 2009;63(1):72-79.

19. Peirano G, van Greune $\mathrm{CH}$, Pitout JD. Characteristics of infections caused by extended-spectrum $\beta$-lactamase-producing Escherichia coli from community hospital in South Africa. Diagn Microbiol Infect Dis. 2011;69(4):449-453. 